\title{
CAN OOCYTES REPAIR FRAGMENTED DNA OF SPERMATOZOA?
}

\author{
Michal Ješeta ${ }^{1,5 *}$, Markéta Myšková ${ }^{2}, J^{2}$ ana Žáková ${ }^{1}$, Igor Crha ${ }^{1,3}$, Karel Crha ${ }^{1}$, Eva Chmelikova ${ }^{5}$, \\ Elena Kistanova ${ }^{4}$, Pavel Ventruba ${ }^{1}$
}

\begin{abstract}
Approximately half of the cases of infertility are due to male factor. In many cases the underlying cause of male infertility is not discovered and, therefore, the condition is considered idiopathic. Examination of morphology, motility, concentration and total sperm count is very important but not sufficient for complex men fertility evaluation. Sperm DNA integrity is a very important one. Sperm DNA can be fragmented by several internal or external factors. In immature sperm cells, the DNA can be repaired by reparatory mechanisms of spermatogonia or spermatocytes. However, in a haploid mature sperm cell, these fragments can not be repaired by male and the fragmented DNA is transferred to oocyte. Oocytes are able to repair male fragmented DNA after their fertilization. A quality embryo can repair damaged sperm DNA and the repair system depends on cytoplasmic and genomic quality of the oocyte. The ability of oocyte to repair sperm DNA strong depend on quality of fertilized oocytes.
\end{abstract}

Running title: Oocyte and DNA repair

Keywords: spermatozoa, DNA integrity, apoptosis, sperm preparation

\footnotetext{
${ }^{1}$ Department of Obstetrics and Gynecology, Faculty of Medicine, Masaryk University and University Hospital Brno, Brno, Czech Republic

${ }^{2}$ Department of Animal Physiology\& Immunology, Faculty of Science, Masaryk University, Brno, Czech Republic

${ }^{3}$ Department of Nursing and Midwifery, Faculty of Medicine, Masaryk University, Brno, Czech Republic

${ }^{4}$ Institute of Biology and Immunology of Reproduction, Bulgarian Academy of Sciences, Sofia, Bulgaria

${ }^{5}$ Department of Veterinary Sciences, Czech University of Life Sciences in Prague, Czech Republic

* Correspondence: jeseta.michal@fnbrno.cz

Full list of author information is available at the end of article
} 


\section{Introduction}

Spermatozoa are extremely sensitive cells and in recent years, male infertility deterioration has been recorded globally [1]. Examination of male infertility factor is represented mainly by a complete semen analysis. Nevertheless, examination of morphology, motility, concentration and total sperm count is not sufficient nowadays and other sperm parameters are observed more and more often. Despite being protected from adverse effects at the beginning of spermatogenesis, sperm DNA can be significantly fragmented in several ways. Sperm cells and their DNA are adversely affected not only by internal factors, but also by environmental factors. The internal factors include formation of aberrations during recombination, abnormal maturation of spermatids and abnormal protamination, apoptosis and oxidative stress [2]. Environmental factors affecting DNA are age, ejaculatory abstinence interval, heat stress, some medications and treatment procedures, pollutants, chemotherapy, smoking, varicocele, leucospermia and processes of cryoconservation [2]. Maintaining the sperm DNA compact and intact is essential for fertilization, formation of zygote and proper development of embryo. It is evident now, that spermatozoa with damaged DNA are able to reach the place of fertilization in vivo, to fertilize oocytes and generate early embryos in both in vivo and in vitro conditions. Fertilization by a sperm cell with fragmented DNA most often does not manifest on embryo development until the third day of cleavage, when the paternal genome begins to take effect and embryos may begin to fragment, their development slows down, they do not implant, or early abortions may occur after transfer of such embryos.

\section{Protection from fragmentation}

In its final form, sperm DNA is packed in special basic proteins called protamines. They provide more reliable protection of genetic information than histones, forming a part of chromatin in somatic cells. The protamines are basic and positively charged proteins [3]. They have these qualities due to their $-\mathrm{NH}^{3}+$ and $-\mathrm{SH}$ groups and also due to their amino acid composition. The most abundant amino acid in protamines is arginine, which makes up to $45-48 \%$ of the total molecule structure [4]. The positively charged $-\mathrm{NH}^{3}+$ groups of arginine neutralize the negatively charged phosphate groups of DNA, which allows formation of strong bonds and correct chromatin condensation [5]. Other abundant amino acids are histidine and cysteine with thiol groups (-SH) [6]. There are also amino acids serine and threonine, which can also bind negatively, charged phosphate groups. If these amino acids are dephosphorylated, unpacking of the DNA occurs. This happens after fertilization of an oocyte during formation of paternal pronucleus [7]. Protamines are therefore essential not only for sperm maturation, but they also play an important role during oocyte fertilization [8].

Thanks to a higher positive charge of protamines, the DNA is more condensed, so that its volume is only one-sixth of the DNA volume in somatic cells. This structure does not contain water, it is crystalline, which is very important in the issue of DNA disruption [7].

The protamines are coded by PRM1 and PRM2 genes. Both the genes are located on 16p13.3 chromosome and are transcribed in early phases of spermatogenesis in postmeiotic spermatids [9]. In the spermatids, mRNA of PRM1 and PRM2 genes is present, but not protamine 1 and 2 proteins themselves. Translation of the mRNA of PRM1 and PRM2 genes is not initiated until spermatids elongation phase [9].

The DNA is gradually packaged on protamines and due to this alternation, the chromatin in sperm nucleus achieves its final form and sperm DNA can be better protected from adverse chemical and physical factors. On the other side, during spermatogenesis, storage of spermatozoa and their transfer, it means when DNA is bound to protamines, its repair is disabled [3]. Despite reparation mechanisms, also transcription activity is ceased during protamination [10].

These proteins are not the only insurance of correct structure and function of DNA. Other mechanisms of sperm genetic material protection are strengthening of bonds in chromatin with zinc ions and ability of seminal plasma to reduce the negative impact of oxygen species.

Zinc is an important stabilizing element of sperm DNA. Zinc ion $\left(\mathrm{Zn}^{2}+\right)$ deficiency or loss is accompanied with infertility. Zinc ions are incorporated in sperm cells during compaction of nucleus and if zinc is not present, spermatogenesis is ceased [11]. Due to the fact that zinc is not subject to oxidation, it probably protects thiol groups from degradation and from their premature binding by disulphide bonds [6].

During oocyte fertilization, zinc from paternal DNA in sperm is eliminated after transition to ooplasm and sperm DNA strands are dissociated subsequently [12]. However, if zinc is eliminated during oocyte fertilization while chromatin repulsion does not occur at the same time and its fibers are not separated, free thiol groups can be oxidized into disulphide bonds. This can affect transfer of sperm DNA within oocyte, at the same time it can lead to development of a defective zygote. Even premature or partial elimination of zinc is harmful to sperm and sperm DNA becomes more accessible for negative factors with damaging effect on the DNA [12].

Chromatin integrity in ejaculated sperm cells depends on zinc. Therefore, other zinc ions are absorbed from prostate secretions during ejaculation. For this reason, the concentration of zinc in ejacu- 
lated spermatozoa is higher than in the spermatozoa stored in epididymis [13].

Anti-oxidative protection of sperm cells by seminal plasma is another way of protection from DNA fragmentation. Seminal plasma contains low-molecular and high-molecular anti-oxidative factors. Those with the highest molecular weight are superoxide dismutase, glutathione peroxidase and catalase [14]. The anti-oxidants with low molecular weight are less important and contain taurine and hypoxitaurine present in seminal plasma, protecting membranes of sperm cells from lipid peroxidation [15].

Anti-oxidativecapacity is associated with the level of sperm motility and whether its morphology will be normalor abnormal. In men with sperm pathology (lower motility, worse morphology, lower count), the anti-oxidative capacity of both sperm cells and seminal plasma was often significantly decreased [14].

\section{Repair of DNA breaks}

Although the systems mentioned above protect sperm DNA from damage, its fragmentation may occur and often does occur. If this happens in immature sperm cells, the DNA is repaired by intensive and efficient reparatory mechanisms of spermatogonia or spermatocytes. In a haploid mature sperm cell, these errors cannot be repaired anymore and the fragmented DNA is transferred to oocyte [16]. If the reparatory system of the oocyte fail as well, zygote and subsequent embryos with chromosomal abnormalities are formed [17].

Cells utilize several mechanisms for reparation of DNA errors occurring during their development and life. The first one is the direct reversal of damage, DDR, which is very important for proper development of oocyte and early embryo. This mechanism uses enzymes for reparation of incorrectly methylated bases. In oocytes, this is a key reparatory mechanism, since methylation is very important during gene imprinting [18].

Another mechanism is the single-strand damage repair (SSR) for the single strand breaks. For reparations of double-strand breaks there is the double-strand breaks repair (DSBs). A breakage in both strands is the most serious type of DNA damage. The DSBs mechanism has two subtypes, the non-homologous end joining; NHEJ and the homologous recombination (HR). The HR is active in later phases of cell cycle, i.e. in the $S$ and G2 phase [19]. It is a part of checkpoints G1/S and G2/M, where it repairs errors occurring during cell division. The NHEJ shows activity throughout the cell cycle, however the highest activity is recorded in the G1 phase [18].

If only one base is damaged, the base excision repair (BER) is used which repairs the DNA by excision of a single base [18].However, there are also errors where larger parts of DNA need to be excised, the nucleotide excision repair (NER) is used in such cases. This mechanism can excise a part of up to 30 incorrect nucleotides from the DNA. Such errors can already change the shape of the helix. In an egg, there are two subtypes of this repair mechanism - the global genomic NER; GG-NER) and the transcription coupled NER; TC-NER), that can search for errors in the helix by various methods. The TC-NER repairs faster than the GG-NER and starts repairing immediately when the RNA polymerase stops [18].

The last repair mechanism is the mismatch repair (MMR), a system repairing errors in base pairing ( $\mathrm{G} / \mathrm{T}$ and $\mathrm{A} / \mathrm{C}$ pairs are formed) or in wrong nucleotidesequencing. The process can repair a DNA sequence with wrong pairing of up to 1000 base pairs. This incorrect pairing often occurs during synthesis. The reparation mechanism is triggered when an anomaly is found on the tertiary structure [18].

\section{Sperm DNA repair by oocyte reparatory mechanisms}

Sperm cells are able to repair their DNA only at the beginning of their development, particularly until the transcription and translation are finished. At the moment when morphologically mature sperm cell is transported for storage in epididymis, or is a part of ejaculate, it is no longer able to repair incorrect DNA. At this time, the only possibility to repair fragmented sperm DNA is by the reparatory mechanisms of an oocyte or early embryo. A quality embryo can repair damaged sperm DNA and the repair system depends on cytoplasmic and genomic quality of the oocyte [18]. To some extent, this prevents transfer of paternal aberrations to the DNA of embryo, if the oocytes are of a good quality, as in young patient and cycles with oocyte donation, when the donors of fertilized eggs are young healthy women. Besides that, it is well known that endogenous and exogenous damage of DNA occurs immediately after oocyte fertilization. Fully functional repair system of oocyte DNA is able to correct a persistent unrepaired damage in both maternal and paternal genome [18]. In case when all mistakes are repaired, even a sperm cell with fragmented DNA can create a healthy offspring [16]. However, much more common situation is when errors in sperm DNA are not repaired by oocyte nor by early embryo. Then, the embryo does not implant or is aborted. But a situation may occur when defective paternal DNA is transferred to an oocyte, the repair mechanisms are not sufficient and an embryo with genetically determined developmental defects is created [16]. It has been documented, that human oocytes have high expression of DNA repair genes [18].

In somatic cells, up to thousands of DNA errors develop in one day and they need to be repaired in shortest time. Numerous errors occur not only in somatic cells, but also in embryos, in the first 24 hours of development. A cell can resolve a presence of DNA damage by three different ways - by initia- 
tion of apoptosis, by tolerating the mistakes, which can lead to mutations, or by repair of the DNA errors [18]. In embryonic cells, the cell cycle is much shorter than in adult cells. Genome integrity is therefore in higher risk during embryo development and DNA repair efficiency in these early stages is crucial for further development.

In repair of sperm DNA breaks, the mechanism repairing errors in one strand are employed the most, i. e. BER, NER and MMR mechanisms [20]. They repair DNA by excision of incorrect basis, nucleotide or by correction of wrong pairing. It is generally easier and more efficient to repair breaks on one strand of the DNA helix than breaks on both strands [21]. State that oocytes have ability to repair sperm DNA, if less than $8 \%$ of paternal DNA is damaged. Gene expression of these reparatory mechanisms differs in oocyte developmental stages and differsalso in women of different ages. In younger women, expression of these and also other reparatory mechanisms is significantly higher than their expression in older women [22].

\section{Ability of oocytes to repair sperm DNA in older women}

In older women, ability to repair DNA is disrupted due to decreased expression of genes employed in the reparatory process [23]. This theory was confirmed by research of Horta et al. [22] who observed 30 mouse females of different age. First group included young mice immediately after reaching sexual maturity, in the second group the mice were significantly older. Oocytes of both mice groups were fertilized with sperm cells that were purposefully exposed to radiation. The radiation caused disruption of sperm DNA integrity. The experiment showed that oocytes of older mice had significantly lower expression of the genes involved in DNA reparatory mechanisms and they were not able to repair damaged DNA after fertilization with sperm with fragmented DNA. These fertilized oocytes developed into zygotes and two-cell embryos with lower activity of the DDR mechanism. A similar phenomenon probably works also in human, whose expected age limit is 35 years, after that the DNA repair activity in oocytes decreases [24]. Ability of a human embryo to react to damaged DNA and repair it and its selective sensitivity to a specific damage are still not studied sufficiently. There are many gaps in the current knowledge on exact roles and expression timing of several genes for DNA repair in early stages of embryonic development. The observed development-specific variations in expression transcripts of genes for reparation of proteins and DNA indicate how complex regulation of these processes is during development.

In cases of woman infertility associated with age, repeated pregnancy failures after repeated treatment with assisted reproduction and absence of male infertility factors, the program of oocyte donorship could be and often is a way to improve success of implantation and pregnancy [25]. Recent research has revealed that when oocyte of young women from egg donor cycles are fertilized, the impact of DNA integrity damage level on embryo development was minimal. The ability of oocyte to repair sperm DNA seems to be really high and good quality oocytes can repair damaged paternal DNA very efficiently [26].

\section{Conclusions}

Fragmented DNA is limiting factor negatively affecting embryonal development. The human oocyte is relatively competent to repair DNA damage after fertilization. However, repair activity in oocyte or zygote is affected many unclear factors. For example, RNA expression is very important but not all mRNA for DNA repair are fully translated. Part mRNA is translated rapidly after fertilization and must be protected and stored for later stage of embryonal development. For next progress in this field, we will need more information regarding of these mechanisms in mammalian and primary in human oocytes.

\section{Ethical approval}

The conducted research is not related to either human or animal use.

\section{Acknowledgements}

Publication of this article was made possible by grant Czech Ministry of Education, Youth and Sports MSMT LTC 18059.

\section{Corresponding author}

Ješeta Michal, Department of Obstetrics and Gynecology, University Hospital and Masaryk University, Obilni trh 11, 60200 Brno, Czech Republic, e-mail address: jeseta.michal@fnbrno.cz.

\section{Conflict of interest statement}

The authors declare they have no conflicts of interest.

\section{References:}

1. Levine H, Jørgensen N, Martino-Andrade A, Mendiola J, Weksler-Derri D, Mindlis I, Pinotti R., Swan SH. Temporal trends in sperm count: A systematic review and meta-regression analysis. Hum Reprod Update. 2017;23(6):646-59. DOI:10.1093/humupd/dmx022.

2. Gunes S, Sertyel S. Sperm DNA Damage and Oocyte Repair Capability. A Clin Guid to Sperm DNA Chromatin Damage. 2018;152(1):321-46. DOI:10.1007/978-3-319-71815-6_18.

3. ZiniA, Agarwal A, editors. Sperm chromatin: biological and clinical applications in male infertility. New York: Springer International Publishing; $2011.512 \mathrm{p}$.

4. Gusse M, Sauti P, Baiche D, Martinage A, Roux C, Dadoune JP, Chevaillier P. Purification and characterization of nuclear basic proteins of human sperm Electron microscopy. Biochim Biophys Acta - Gen Subj. 1986;884(1):124-34. DOI:10.1530/REP-16-0077.

5. Balhorn R. The protamine family of sperm nuclear proteins. Genome Biol. 2007;8(9):227. DOI:10.1186/gb-2007-8-9-227.

6. Kvist U, Afzelius B, Nilsson L. The intrinsic mechanism of chromatin decondensation and its activation in human spermatozoa. 1980;22(3):543-54. DOI:10.1111/j.1440-169X.1980.00543.x.

7. Baldi E, Muratori M, editors. Genetic Damage in Human Spermatozoa New York: Springer International Publishing; 2013.194 p.

8. Zhao Y, Li Q, Yao C, Wang Z, Zhou Y, Wang Y, Liu L., Wang Y., Wang L., Qiao Z. Characterization and quantification of mRNA transcripts in ejaculated spermatozoa of fertile men by serial analysis of gene expression 2006;21(6):1583-90. DOI:10.1093/humrep/del027. 
9. Hecht NB, Kleene KC, Yelick PC, Johnson PA, Pravtcheva DD, Ruddle FH. Mapping of haploid expressed genes: Genes for both mouse protamines are located on chromosome 16. Somat Cell Mol Genet. 1986;12(2):2038.DOI:10.1007/BF01560667.

10. Kaur Gill-Sharma M, Choudhuri J, D'Souza S. Sperm Chromatin Protamination: An Endocrine Perspective. Protein Pept Lett. 2012;18(8):786801. DOI:10.2174/092986611795714005.

11. Barney GH, Orgebin-Crist MC, Macapinlac MP. Genesis of Esophageal Parakeratosis and Histologic Changes in the Testes of the Zinc-deficient Rat and Their Reversal by Zinc Repletion. J Nutr. 1968;95(4):526-34. DOI:10.1093/jn/95.4.526

12. Björndahl L, Kvist U. Human sperm chromatin stabilization: A proposed model including zinc bridges. Mol Hum Reprod. 2009;16(1):23-9. DOI:10.1093/molehr/gap099.

13. Kvist U, Bjorndahl L, Roomans GM, Lindholmer C. Nuclear zinc in human epididymal and ejaculated spermatozoa. Acta Physiol Scand. 1985;125(2):297-303. DOI:10.1111/j.1748-1716.1985.tb07719.x.

14. Smith R, Vantman D, Ponce J, Escobar J. Total antioxidant capacity of human seminal plasma. Hum Reprod. 1996;11(8):1655-60. DOI:10.1093/ oxfordjournals.humrep.a019465.

15. Alvarez JG, Storey BT. Role of Superoxide Dismutase in Protecting Rabbit Spermatozoa from 02 Toxicity Due to Lipid Peroxidation. Biol Reprod. 1983;28(5):1129-36. DOI:10.1095/biolreprod28.5.1129.

16. González-Marín C, Gosálvez J, Roy R. Types, Causes, Detection and Repair of DNA Fragmentation in Animal and Human Sperm Cells. Int J Mo Sci. 2012;13(12):14026-52. DOI:10.3390/ijms131114026.

17. Zenzes MT, Puy LA, Bielecki R. Immunodetection of benzo [a ] pyrene adducts in ovarian cells of women exposed to cigarette smoke. 1998;4(2):159-65. DOI:10.1093/molehr/4.2.159.

18. Ménézo Y, Dale B, Cohen M. DNA damage and repair in human oocytes and embryos: A review. Zygote. 2010;18(4):357-65. DOI:10.1017/ S0967199410000286.

19. Rothkamm K, Krüger I, Thompson LH, Löbrich M. Pathways of DNA Double-Strand Break Repair during the Mammalian Cell Cycle. Society. 2003;23(16):5706-15. DOI:10.1128/mcb.23.16.5706-5715.2003.

20. Gardner DK, Sakkas D, Seli E, Wells D , editors. Human Gametes and Preimplantation Embryos. New York: Springer; 2013. 321 p.

21. Ahmadi ALI, Ng S. Fertilizing Ability of DNA-Damaged Spermatozoa 1999;(June 1998):696-704. DOI:10.1002/(sici)1097-010x(19991101) 284:6<696::aid-jez11>3.0.co;2-e.

22. Horta F, Catt S, Ramachandran P, Vollenhoven B, Temple-Smith P. Female ageing affects the DNA repair capacity of oocytes in IVF using a controlled model of sperm DNA damage in mice. Hum Reprod. 2020;35(3):529-44. DOI:10.1093/humrep/dez308.

23. Liu L, Keefe DL. Defective cohesin is associated with age-dependent misaligned chromosomes in oocytes. Reprod Biomed Online [Internet]. 2008;16(1):103-12. Available from: http://dx.doi.org/10.1016/ S1472-6483(10)60562-7.

24. Cimadomo D, Fabozzi G, Vaiarelli A, Ubaldi N, Ubaldi FM, Rienzi L. Impact of maternal age on oocyte and embryo competence. Front Endocrinol (Lausanne). 2018;9(327). DOI:10.3389/fendo.2018.00327.

25. Marchetti F, Essers J, Kanaar R, Wyrobek AJ. Disruption of maternal DNA repair increases sperm-derived chromosomal aberrations. 2016;104(45):17725-9. DOI:10.1073/pnas.0705257104.

26. Antonouli S, Papatheodorou A, Panagiotidis Y, Petousis S, Prapas N. The impact of sperm DNA fragmentation on ICSI outcome in cases of donated oocytes. Arch Gynecol Obstet [Internet]. 2019;300(1):207-215 Available from: https://doi.org/10.1007/s00404-019-05133-9. 